\title{
Supplementation of sodium butyrate protects mice from the development of non-alcoholic steatohepatitis (NASH)
}

\author{
Cheng Jun Jin, Cathrin Sellmann, Anna Janina Engstler, Doreen Ziegenhardt and Ina Bergheim* \\ Institute of Nutritional Sciences, SD Model Systems of Molecular Nutrition, Friedrich-Schiller-University Jena, \\ O7743 Jena, Germany \\ (Submitted 9 April 2015 - Final revision received 16 July 2015 - Accepted 19 August 2015 - First published online 9 October 2015)
}

\section{Abstract}

Overnutrition, insulin resistance and an impaired intestinal barrier function are discussed as critical factors in the development of nonalcoholic fatty liver disease. Not only butyrate-producing probiotics as well as supplementation of sodium butyrate (SoB) have been suggested to bear protective effects on liver damage of various aetiologies. However, whether an oral consumption of SoB has a protective effect on Western-style diet (WSD)-induced non-alcoholic steatohepatitis (NASH) and if so molecular mechanism involved has not yet been determined. Eight-week-old C57BL/6J mice were pair-fed either a liquid control or WSD $\pm 0.6 \mathrm{~g} / \mathrm{kg}$ body weight SoB. After 6 weeks, markers of liver damage, inflammation, toll-like receptor (TLR)-4 signalling, lipid peroxidation and glucose as well as lipid metabolism were determined in the liver tissue. Tight junction protein levels were determined in the duodenal tissue. SoB supplementation had no effects on the body weight gain or liver weight of WSD-fed mice, whereas liver steatosis and hepatic inflammation were significantly decreased (e.g. less inflammatory foci and neutrophils) when compared with mice fed only a WSD. Tight junction protein levels in duodenum, hepatic mRNA expression of TLR-4 and sterol regulatory element-binding protein 1c were altered similarly in both WSD groups when compared with controls, whereas protein levels of myeloid differentiation primary response gene 88, inducible nitric oxide synthase, 4-hydroxynonenal protein adducts and F4/80 macrophages were only significantly induced in livers of mice fed only the WSD. In summary, these data suggest that an oral supplementation of SoB protects mice from inflammation in the liver and thus from the development of WSD-induced NASH.

Key words: Non-alcoholic fatty liver disease: Sodium butyrate: Hepatic inflammation: Inducible nitric oxide synthase: Reactive oxygen species

During the last decades, the prevalence of non-alcoholic fatty liver disease (NAFLD), comprising a wide spectrum of liver diseases ranging from simple hepatic steatosis to non-alcoholic steatohepatitis (NASH), cirrhosis and even hepatocellular carcinoma $^{(1)}$, has dramatically increased. Indeed, Blachier et $a l^{(2)}$ has recently reported in a survey reviewing 260 epidemiological studies published in Europe during the last 5 years that NAFLD is by now the most frequent liver disease in Europe. However, in spite of intensive research efforts during the last decades, molecular mechanisms underlying the development of NAFLD remain mostly unclear, and universally accepted treatment strategies besides a life-style modification, such as weight-reduction diets and/or increases in physical activity are not yet available.

Butyrate, a naturally occurring SCFA in the body mainly produced by intestinal bacteria and found in various foods like cheese and butter, has in recent years been suggested to play an important role in maintaining intestinal homoeostasis ${ }^{(3)}$. Indeed, it has been shown in in vitro studies that butyrate through AMP-kinase- and p38 mitogen-activated protein kinase-dependent signalling cascades may ameliorate ethanolinduced intestinal barrier dysfunction ${ }^{(4,5)}$. Recent animal studies further suggest that sodium butyrate ( $\mathrm{SoB}$ ) may also possess protective effects on liver damage of various aetiologies ${ }^{(6-8)}$. For instance, it has been shown that rats treated before or at the onset of ischaemia reperfusion (I/R) with butyrate were markedly protected from I/R-induced liver injury not only through mechanisms involving inhibition of histone deacetylase and heat shock protein 70 induction in the liver as well as by maintaining the intestinal barrier structure ${ }^{(8)}$. In line with these findings, Cresci et $a l .{ }^{(9)}$ reported that pretreatment of mice with tributyrin, an ester composed of butyric acid and glycerol, protects mice from acute but not chronic alcohol-induced liver damage through mechanisms involving a protection against the

Abbreviations: 4-HNE, 4-hydroxynonenal; ALT, alanine aminotransferase; CCL-2, chemokine (C-C motif) ligand 2; CD8 $\alpha$, cluster of differentiation $8 \alpha$; FAS, fatty acid synthase; iNOS, inducible nitric oxide synthase; IR, insulin receptor; IRS-1, IR substrate 1; I/R, ischaemia reperfusion; MyD88, myeloid differentiation primary response gene 88 ; NAFLD, non-alcoholic fatty liver disease; NASH, non-alcoholic steatohepatitis; SoB, sodium butyrate; SREBP-1c, sterol regulatory element-binding protein 1c; TGF- $\beta 1$, transforming growth factor beta 1; TLR-4, toll-like receptor 4; WSD, Western-style diet; ZO-1, zona occludens protein 1.

* Corresponding author: I. Bergheim, fax +49 3641 949632, email ina.bergheim@uni-jena.de 
loss of tight junction proteins induced by acute alcohol exposure. Furthermore, Mattace et al. ${ }^{(6)}$ showed that butyrate and its synthetic derivative $N$-(1-carbamoyl-2-phenyl-ethyl) butiramide may protect rats from the development of insulin resistance and NAFLD. However, not only whether butyrate also possesses protective effects on the development of NAFLD in other models of NAFLD, as well as the molecular mechanisms involved has not yet been determined.

Using a mouse model in which mice were pair-fed a fat, fructose and cholesterol-enriched liquid diet to induce NAFLD, the main objective of the present study was to test the hypothesis that an oral supplementation of $\mathrm{SoB}$ protects mice from the development of NASH and if so, to determine underlying molecular mechanisms involved.

\section{Methods}

\section{Animals and treatments}

Eight-week-old female C57BL/6J mice (Janvier SAS), which were indicated to be more susceptible to a fructose-induced NAFLD compared with the male mice by our own group ${ }^{(10)}$, were housed in a pathogen-free barrier facility accredited by the Association for Assessment and Accreditation of Laboratory Animal Care. The local Institutional Animal Care and Use Committee approved all procedures. During a 6-week feeding period, mice ( $n$ 6/group) were pair-fed either a standard liquid diet (control, $15.7 \mathrm{MJ} / \mathrm{kg}$ diet: $69 \mathrm{E} \%$ from carbohydrates, $12 \mathrm{E} \%$ from fat and $19 \mathrm{E} \%$ from protein) or a liquid Western-style diet (WSD; fortified with fructose, fat and cholesterol; $17.8 \mathrm{MJ} / \mathrm{kg}$ diet: $60 \mathrm{E} \%$ from carbohydrate, $25 \mathrm{E} \%$ from fat and $15 \mathrm{E} \%$ from protein with $50 \%, \mathrm{w} / \mathrm{w}$ fructose and $0.155 \%, \mathrm{w} / \mathrm{w}$ cholesterol) (Ssniff) $\pm 0.6 \mathrm{~g} / \mathrm{kg}$ body weight $/ \mathrm{d}$ SoB (Sigma-Aldrich) (for details also see online Supplementary Table S1). All mice had free access to plain tap water. Diet consumption was assessed and adjusted daily, and body weight was registered weekly. After 4 weeks of feeding, mice were fasted for $6 \mathrm{~h}$ to obtain fasting blood samples from retrobulbar venous plexus for glucose measurements. After 6 weeks, mice were anaesthetised with the mixture solution of $100 \mathrm{mg}$ ketamine/ $\mathrm{kg}$ and $16 \mathrm{mg}$ xylazine/kg body weight by intraperitoneal injection. Blood was collected just before killing. Liver and intestine samples were either fixed in neutral-buffered formalin or in optimal cutting temperature (OCT) embedding media (Medite) for the histological staining or frozen immediately in liquid $\mathrm{N}_{2}$ and were then kept in a $-80^{\circ} \mathrm{C}$ freezer for the further experiments.

\section{Histological evaluation of liver sections}

Frozen sections of the liver fixed in OCT $(10 \mu \mathrm{m})$ were stained with Oil red $O$ (Sigma) as described previously ${ }^{(11)}$. Representative photomicrographs were captured at a $100 \times$ magnification using a system incorporated in microscope (Leica DM4000; Leica). Paraffin-embedded liver sections ( $4 \mu \mathrm{m})$ were stained with haematoxylin-eosin (Sigma) to evaluate histology. Representative photomicrographs were captured using a camera integrated in a microscope (Leica DM4000 B LED; Leica) at 200× magnification. NAFLD activity score was used to determine liver damage ${ }^{(12)}$. To determine number of neutrophilic granulocytes, liver sections $(4 \mu \mathrm{m})$ were stained with Naphthol AS-D Chloroacetate Esterase kit (Sigma), and number of neutrophils was determined as previously described in detail ${ }^{(11)}$. Collagen deposition in the liver tissue was stained with Picrosirius red (Sigma) and counterstained with fast green (Sigma) to determine fibrosis. Quantitive evaluation of staining was carried out as previously described in detail ${ }^{(13)}$.

\section{Blood parameter of liver damage and ELISA}

Alanine aminotransferase (ALT) activity was determined by a colorimetric reaction (Architect, Fa.; Abbott) in a routine laboratory. Fasting blood glucose levels were measured using a blood glucose meter (Contour; Bayer Vital GmbH). Protein concentration of TNF- $\alpha$ in the liver tissue was determined using a commercially available ELISA kit (Mouse TNF-alpha ELISA Kit; Assaypro) as detailed before ${ }^{(14)}$.

\section{Immunohistochemical staining of the liver and duodenum}

Paraffin-embedded liver tissue sections $(4 \mu \mathrm{m})$ were stained for myeloid differentiation primary response gene 88 (MyD88), 4-hydroxynonenal (4-HNE) protein adducts, inducible nitric oxide synthase (iNOS), F4/80 and cluster of differentiation $8 \alpha$ (CD8 $\alpha$ )-positive cells using polyclonal antibodies (MyD88: Santa Cruz Biotechnology; 4-HNE: AG Scientific; iNOS: Affinity BioReagents; F4/80: Abcam; CD8 $\alpha$ : Santa Cruz Biotechnology) as previously detailed ${ }^{(15,16)}$. Paraffin-embedded duodenal tissue sections $(4 \mu \mathrm{m})$ were stained for the tight junction proteins occludin and zonula occludens 1 (ZO-1) using polyclonal primary antibodies (Life Technologies $\mathrm{GmbH}$ ) as described previously ${ }^{(11,17)}$. In brief, to detect the binding of target protein to the specific primary antibody, tissue sections were incubated with a peroxidase-linked secondary antibody and diaminobenzidine (Peroxidase Envision Kit; Dako). Pictures of eight fields of each tissue section ( $\times 200$ of each liver tissue; $\times 400$ of each duodenum tissue) were captured using a digital camera integrated in a microscope (Leica DM4000 B LED), and the extent of staining in tissue sections was defined as per cent of field area within the default colour range determined by an analysis system incorporated in microscope. Mean values of eight sections were used per tissue section to determine means.

\section{RNA isolation and real-time RT-PCR}

Total RNA from the liver tissue was isolated with Trizol (peqGOLD Trifast; Peqlab), and $1.0 \mu \mathrm{g}$ total RNA was reverse transcribed (cDNA synthesis kit; Promega). SYBR Green ${ }^{\circledR}$ Supermix (Agilent Technologies) was used to prepare the PCR mix, and relative mRNA expression was determined using a MX QPCR System (Agilent Technologies) as previously detailed ${ }^{(14)}$. PCR primers for chemokine (C-C motif) ligand 2 (CCL-2), toll-like receptor $4(T L R-4)$, insulin receptor $(I R)$, IR substrate 1 $(I R S-1), I L-1 \beta$, fatty acid synthase (FAS), sterol regulatory element-binding protein 1c (SREBP-1c), transforming growth factor $\beta 1(T G F-\beta 1)$ and eukaryotic translation elongation factor 2 (Eef-2) as well as $18 S$ were designed using Primer 3 software 
(Whitehead Institute for Biomedical Research). Sequences are listed in Table 1 . The comparative cycle threshold $\left(C_{t}\right)$ method was used to determine the amount of target, normalised to an endogenous reference (Eef-2) and relative to a calibrator $\left(2^{-\Delta \Delta C_{t}}\right)$. The purity of the PCR products was verified by melting curves and gel electrophoresis.

\section{Western blot}

A Dignum A buffer ( $1 \mathrm{~mol} / 1 \mathrm{HEPES}, 1 \mathrm{~mol} / 1 \mathrm{MgCl}_{2}, 2 \mathrm{~mol} / \mathrm{l} \mathrm{KCl}$, $1 \mathrm{~mol} / \mathrm{l}$ dithiothreitol) containing a protease inhibitor mix (Roche) was used to homogenise liver tissue samples, and blots were prepared as previously detailed ${ }^{(18)}$. Blots were probed with antibodies against inhibitor of NF- $\kappa \mathrm{B}$ kinase (IKK) subunit $\beta$ and phosphorylated IKK subunit $\alpha$ and $\beta$ (Ser 176/ 180) $(\mathrm{pIKK} \alpha / \beta)$ (both Cell Signaling Technology), and bands were visualised using a Super Signal Western Dura kit (Thermo Scientific). Equal loading of blots was ensured by Ponceau Red staining (Roth). Using a ChemiDoc MP System (Bio-Rad Laboratories), protein bands were detected and analysed.

\section{Statistical analysis}

All data were presented as means values with their standard errors. One-way ANOVA with Tukey's post hoc test was used to determine the statistical differences between the treatment groups (Graph Pad Prism version 6.0; GraphPad Software). Outliers were identified using Grubbs's test. $P$ value $<0.05$ was considered to be significant.

\section{Results}

\section{Effect of an oral sodium butyrate supplementation on liver} injury and markers of glucose metabolism

As expected, mice fed the WSD developed massive macrovesicular steatosis associated with marked inflammatory alterations (see Fig. 1). In contrast, despite a similar energy intake, body weight gain and even a significantly higher liver: body weight ratio, mice fed a WSD supplemented with SoB (WSD + SoB) had a significantly lower number of fat infiltrated hepatocytes and displayed predominantly microvesicular fat accumulation in the liver (see Table 2, Fig. 1(a)-(d)). However, number of fat infiltrated hepatocytes in the WSD + SoB group was still significantly higher than that in both control groups (see Fig. 1(d)). Furthermore, number of not only inflammatory focis as well as neutrophils and F4/80 positive cells were only found to be significantly higher in livers of mice fed a WSD alone $(P<0.05$ in comparison with all other groups) (see Table 3 and Fig. 1(e)). In line with these findings, number of CD8 $\alpha$-positive cells in livers of mice receiving only WSD was also significantly higher than in those of mice fed the control diet or WSD + SoB. However, despite not showing any signs of inflammation, number of $\mathrm{CD} 8 \alpha$-positive cells was significantly higher in livers of mice fed the control diet supplemented with SoB $(\mathrm{C}+\mathrm{SoB}$ ) (see Table 3). Expression of CCL-2 mRNA was only found to be significantly higher in livers of WSD-fed mice when compared with all other groups (see Table 3). TNF- $\alpha$ protein concentration was only by trend higher in livers of mice fed the WSD alone in comparison with control mice $(P=0.065)$ (see Table 3 ). In line with these findings, concentration of $\operatorname{pIKK} \alpha / \beta$ was only significantly higher in mice fed the WSD alone (see Fig. 3(g) and (h)). A similar effect of the WSD feeding was not found in mice concomitantly fed SoB; however, data varied considerably in some groups. Expression of $I L-1 \beta$ mRNA was significantly higher in livers of mice fed only a WSD in comparison with controls; however, expression of $I L-1 \beta$ mRNA was also significantly higher in livers of both groups receiving SoB, irrespective of the diet fed (see Table 3). Sirius red staining of collagen deposition revealed no differences between the different treatment groups (see Table 3 and Fig. 2(a)). Expression of TGF- $\beta 1$ mRNA in the liver of WSD-fed mice was significantly higher than that in both control groups. In livers of mice fed the WSD + SoB, TGF- $\beta 1$ mRNA expression was only significantly higher than that in those of mice fed only the control diet (see Table 3). As mice only displayed the beginning of NASH and measurements varied considerably, ALT plasma levels did not differ between groups (see Table 2). Furthermore, fasting glucose levels did not differ between groups (see Fig. 1(f)). In contrast, expression of $I R$ was markedly higher in livers of mice fed the WSD+SoB; however, as expression varied considerably in the control groups, differences only reached the level of significance for the comparison of the two WSD groups (see Table 2). Furthermore, although IRS-1 expression was significantly lower in livers of mice of all groups in comparison with controls, expression of IRS-1 did not differ between groups treated with SoB, irrespective of the diet fed (see Table 2).

Table 1. Primer sequences used for real-time RT-PCR detection

\begin{tabular}{|c|c|c|}
\hline & Forward $\left(5^{\prime}-3^{\prime}\right)$ & Reverse $\left(5^{\prime}-3^{\prime}\right)$ \\
\hline$C C L-2$ & GCC AGA CGG GAG GAA GGC CA & TGG ATG CTC CAG CCG GCA AC \\
\hline$T L R-4$ & AGC CAT TGC TGC CAA CAT CA & GCT GCC TCA GCA GGG ACT TC \\
\hline IR & CAT CCC GAA AGC GAA GAT CC & GAG TCC TGA TTG CAT GCC TGC \\
\hline IRS-1 & GTT GCC ACC CCT AGA CAA AA & GCT CTA GTG CTT CCG TGT CC \\
\hline$I L-1 \beta$ & TGG CTG TGG AGA AGC TGT GG & GTC CGA CAG CAC GAG GCT TT \\
\hline FAS & TCT GGG CCA ACC TCA TTG GT & GAA GCT GGG GGT CCA TTG TG \\
\hline$S R E B P-1 C$ & ACC GGC TAC TGC TGG ACT GC & AGA GCA AGA GGG TGC CAT CG \\
\hline$T G F-\beta 1$ & GTC TGG GAC CCT GCC CCT AT & TTG CAG GAG CGC ACG ATC AT \\
\hline Eef-2 & GTG ACA GCT GCC TTG CGT GT & GAT GCG CTG GAA GGT CTG GT \\
\hline 185 & GTA ACC CGT TGA ACC CCA TT & CCA TCC AAT CGG TAG TAG CG \\
\hline
\end{tabular}

$C C L-2$, chemokine (C-C motif) ligand 2; TLR-4, toll-like receptor 4; IR, insulin receptor; IRS-1, insulin receptor substrate 1 ; FAS, fatty acid synthase; SREBP-1C, sterol regulatory element-binding protein 1c; TGF- $\beta 1$, transforming growth factor $\beta 1$; Eef-2, eukaryotic translation elongation factor 2. 
(a)

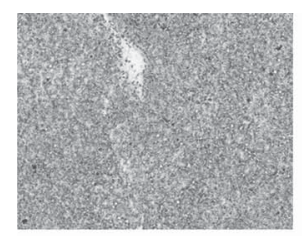

C

(b)

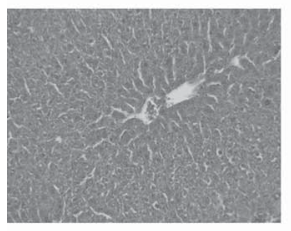

C

(c)

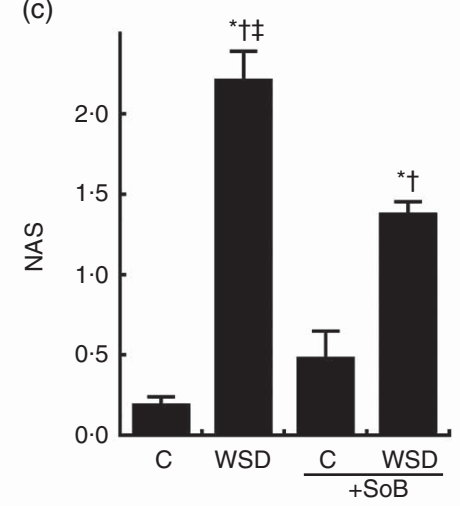

(e)

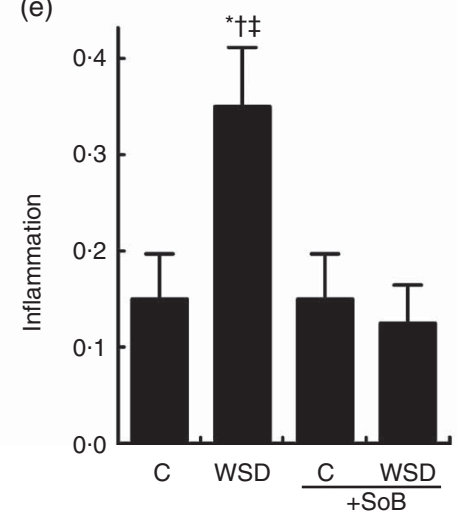

Oil Red O

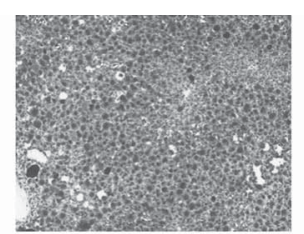

WSD

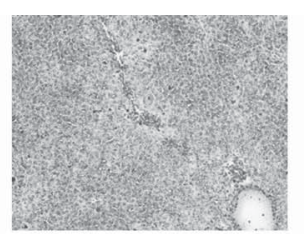

$\mathrm{C}+\mathrm{SoB}$

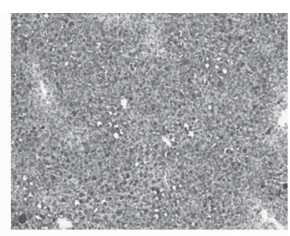

WSD+SoB

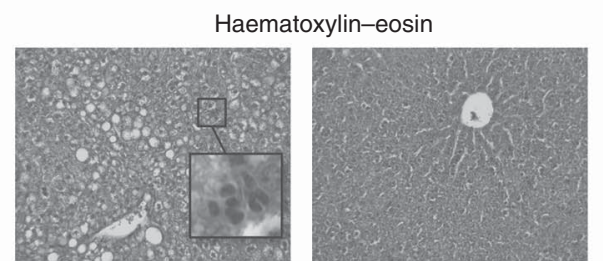

$\mathrm{C}+\mathrm{SoB}$

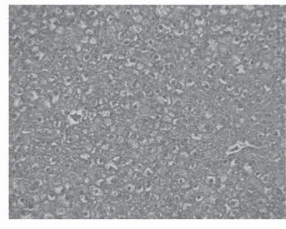

WSD+SoB (d)

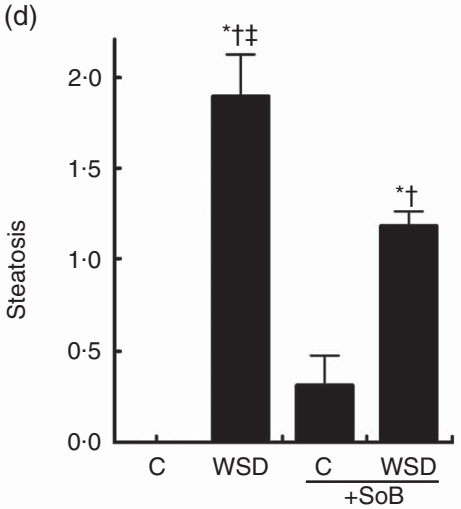

(f)

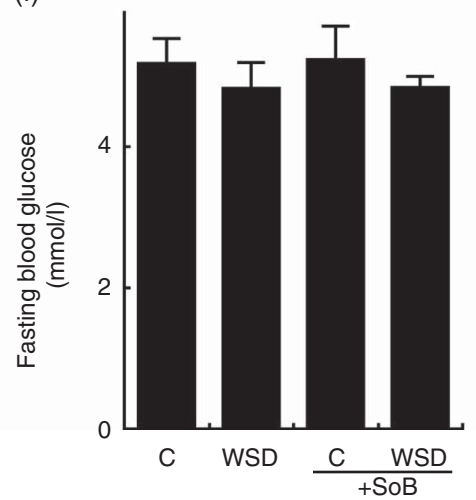

Fig. 1. Effect of an oral supplementation of sodium butyrate (SoB) on the level of steatosis and inflammation as well as the level of fibrosis in the liver of Western-style diet (WSD)- or control diet (C)-fed mice. (a) Representative photomicrographs of Oil Red O staining (upper panel) of liver sections (100x). (b) Representative photomicrographs of haematoxylin-eosin staining (lower panel) of liver sections (200x). (c) Evaluation of liver damage using a non-alcoholic fatty liver disease activity score (NAS). Analysis of hepatic (d) steatosis and (e) inflammation using NAS. (f) Fasting blood glucose levels of mice. Values are means, with their standard errors represented by vertical bars. * $P<0.05$ compared with mice fed control diet, $\dagger P<0.05$ compared with mice fed concomitantly control diet and SoB ( $0.6 \mathrm{~g}$ SoB/kg body weight/d), $¥ P<0.05$ compared with mice fed concomitantly WSD and SoB $(0.6 \mathrm{~g} \mathrm{SoB} / \mathrm{kg}$ body weight/d).

\section{Effect of oral sodium butyrate supplementation on tight} junction protein levels in duodenum and on markers of the toll-like receptor 4 signalling cascade in the liver

As butyrate has been suggested to at least in part mediate its beneficial effects on the development of liver diseases through altering intestinal barrier function ${ }^{(19)}$, we determined protein levels of the tight junction protein occludin and $\mathrm{ZO}-1$ in tissue obtained from duodenum and mRNA expression of the endotoxin receptor TLR-4 as well as its adaptor protein MyD88 in the liver. Protein levels of occludin and ZO-1 were significantly lower in the duodenum of both groups fed a WSD irrespective of additional treatments when compared with both control groups (see Fig. 2(b) and (c), Fig. 3(a) and (b)). Expression of 
Table 2. Effect of an oral supplementation of sodium butyrate (SoB) on body weight, liver:body weight ratio and clinical parameter of liver damage as well as markers of insulin resistance in mice fed a Western-style diet (WSD) or control diet (C)

(Mean values with their standard errors)

\begin{tabular}{|c|c|c|c|c|c|c|c|c|}
\hline & \multicolumn{2}{|c|}{ C } & \multicolumn{2}{|c|}{ WSD } & \multicolumn{2}{|c|}{$\mathrm{C}+\mathrm{SoB}$} & \multicolumn{2}{|c|}{$W S D+S o B$} \\
\hline & Mean & SEM & Mean & SEM & Mean & SEM & Mean & SEM \\
\hline Daily energy uptake (kJ/g of body weight) & $2 \cdot 1$ & 0.17 & $2 \cdot 1$ & 0.04 & 2.01 & 0.04 & 2.01 & 0.04 \\
\hline Daily energy uptake ( $\mathrm{kcal} / \mathrm{g}$ of body weight) & 0.50 & 0.04 & 0.50 & 0.01 & 0.48 & 0.01 & 0.48 & 0.01 \\
\hline Body weight (g) & 21.4 & 0.6 & $22 \cdot 1$ & 0.3 & $21 \cdot 3$ & 0.9 & $22 \cdot 2$ & 0.2 \\
\hline Absolute weight gain (g) & $2 \cdot 4$ & 0.5 & $2 \cdot 6$ & 0.4 & $3 \cdot 1$ & 0.6 & 2.5 & 0.6 \\
\hline Liver weight (g) & 1.1 & 0.0 & $1.4^{*} \ddagger$ & 0.0 & $1 \cdot 1$ & 0.1 & $1 \cdot 4^{*} \ddagger$ & 0.0 \\
\hline Liver:body weight ratio (\%) & 4.9 & 0.1 & $6 \cdot 4^{\star} \ddagger$ & 0.2 & $5 \cdot 1$ & 0.1 & $6 \cdot 2^{*} \ddagger$ & 0.1 \\
\hline ALT (U/I) & $15 \cdot 2$ & 1.0 & 18.9 & 2.4 & 23.1 & 2.9 & $21 \cdot 6$ & $3 \cdot 1$ \\
\hline IR (-fold induction) $\|$ & $2 \cdot 0$ & 0.1 & 1.5 & 0.2 & 1.7 & 0.0 & $2.4 \dagger$ & 0.2 \\
\hline IRS-1 (-fold induction) \| & $4 \cdot 4 \dagger \ddagger \S$ & 0.4 & 2.4 & 0.3 & $2 \cdot 3$ & 0.5 & 2.6 & 0.2 \\
\hline
\end{tabular}

ALT, alanine aminotransferase; IR, insulin receptor; IRS-1, IR substrate 1.

* $P<0.05$ compared with mice fed with control diet.

$\dagger P<0.05$ compared with mice fed with WSD diet.

$\ddagger P<0.05$ compared with mice concomitantly fed control diet and SoB $(0.6 \mathrm{~g} \mathrm{SoB} / \mathrm{kg}$ body weight/d).

$\S P<0.05$ compared with mice concomitantly fed WSD diet and SoB $(0.6 \mathrm{~g} \mathrm{SoB} / \mathrm{kg}$ body weight $/ \mathrm{d})$.

$\| I R$ and IRS-1 mRNA expressions were normalised to Eef-2 mRNA expression.

Table 3. Effect of an oral supplementation of sodium butyrate (SoB) on markers of inflammation and fibrosis in livers of mice fed a Western-style diet (WSD) or control diet (C)

(Mean values with their standard errors)

\begin{tabular}{|c|c|c|c|c|c|c|c|c|}
\hline & \multicolumn{2}{|c|}{ C } & \multicolumn{2}{|c|}{ WSD } & \multicolumn{2}{|c|}{$\mathrm{C}+\mathrm{SoB}$} & \multicolumn{2}{|c|}{ WSD + SoB } \\
\hline & Mean & SEM & Mean & SEM & Mean & SEM & Mean & SEM \\
\hline Neutrophils (number/field) & 1.8 & 0.3 & $4 \cdot 4^{\star} \ddagger \S$ & 0.6 & 1.5 & 0.2 & $1 \cdot 1$ & 0.2 \\
\hline F4/80 (number/field) & $6 \cdot 4$ & 0.7 & $14 \cdot 7^{\star} \neq \S$ & 1.9 & 7.4 & 1.7 & 8.5 & 1.3 \\
\hline CD8a (number/field) & 14.4 & $2 \cdot 8$ & $27 \cdot 6 \star \S$ & $2 \cdot 8$ & $25 \cdot 0 * \S$ & $3 \cdot 2$ & $10 \cdot 1$ & 1.3 \\
\hline CCL-2 (-fold induction) $\|$ & 3.2 & 0.6 & $14 \cdot 6^{\star} \neq \S$ & $4 \cdot 6$ & $2 \cdot 3$ & 0.6 & 3.4 & 0.5 \\
\hline TNF- $a$ (ng/mg protein) & 0.30 & 0.04 & 0.48 & 0.06 & 0.32 & 0.03 & 0.39 & 0.06 \\
\hline$I L-1 \beta$ (-fold induction) $\|$ & $2.5 \dagger \neq \S$ & 0.6 & $6 \cdot 2$ & 0.5 & 4.8 & 0.5 & $6 \cdot 4$ & 0.7 \\
\hline$T G F-\beta 1$ (-fold induction)\| & 1.5 & 0.2 & $3.5^{\star} \ddagger$ & 0.2 & 2.4 & 0.3 & $3 \cdot 2^{*}$ & 0.4 \\
\hline Sirius red (\% per microscopic field) & 0.47 & 0.07 & $0.65^{\top}$ & 0.08 & 0.51 & 0.08 & 0.59 & 0.13 \\
\hline
\end{tabular}

CD8a, cluster of differentiation 8a; CCL-2, chemokine (C-C motif) ligand 2; TGF- $\beta 1$, transforming growth factor $\beta 1$.

${ }^{*} P<0.05$ compared with mice fed with control diet

$\dagger P<0.05$ compared with mice fed with WSD diet.

$\ddagger P<0.05$ compared with mice concomitantly fed control diet and SoB $(0.6 \mathrm{~g} \mathrm{SoB} / \mathrm{kg}$ body weight $/ \mathrm{d})$.

$\S P<0.05$ compared with mice concomitantly fed WSD diet and SoB $(0.6 \mathrm{~g} \mathrm{SoB} / \mathrm{kg}$ body weight/d).

\| CCL-2, IL-1 $\beta$ and TGF- $\beta 1$ mRNA expressions were normalised to $18 S$ mRNA expression.

TLR-4 mRNA was significantly higher in livers of mice fed the WSD alone and in both groups fed diets supplemented with SoB when compared with controls (see Fig. 3(c)). In contrast, protein levels of MyD88 were only significantly induced in livers of mice fed a WSD, whereas MyD88 protein levels of all other groups were at the level of controls (see Fig. 2(d) and Fig. 3(d))

\section{Effect of oral sodium butyrate supplementation on inducible nitric oxide synthase protein levels and markers of lipid peroxidation}

As it has been shown that TLR- 4 mediates its effects also through an activation of iNOS subsequently leading to an increase in the formation of reactive oxygen species ${ }^{(20)}$, we determined iNOS and 4-HNE protein adduct levels in livers of mice. In line with the findings for MyD88, protein levels of iNOS were only found to be significantly induced in mice only fed a
WSD, whereas in livers of those concomitantly treated with SoB while being fed the WSD, protein levels of iNOS were almost at the level of controls (see Fig. 2(e) and Fig. 3(e)). Similar results were also found for levels of 4-HNE protein adducts, also only being significantly higher in livers of mice fed a WSD when compared with controls (see Fig. 3(f)).

\section{Effect of oral sodium butyrate supplementation on markers of hepatic lipid metabolism}

Expression of $S R E B P-1 c$ mRNA in the liver did not differ between WSD groups and was significantly higher than that in livers of control groups (see Fig. 4(a)). In contrast, expression of FAS mRNA was significantly higher in livers of WSD-fed mice when compared with all other groups. However, although being significantly lower in livers of mice fed WSD alone, FAS mRNA expression in livers of WSD + SoB-fed mice was still significantly higher than that in both control groups (see Fig. 4(b)). 
(a)

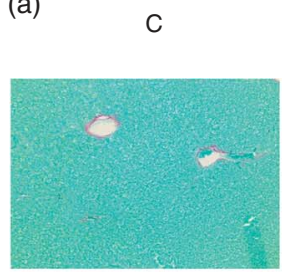

(b)

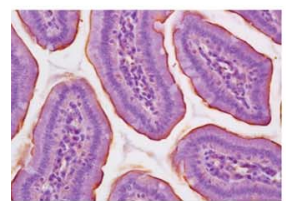

(c)

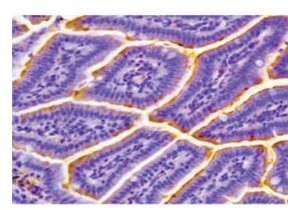

(d)

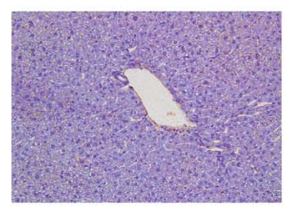

(e)

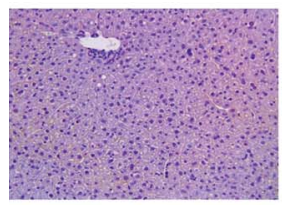

(f)

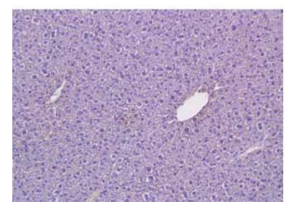

WSD

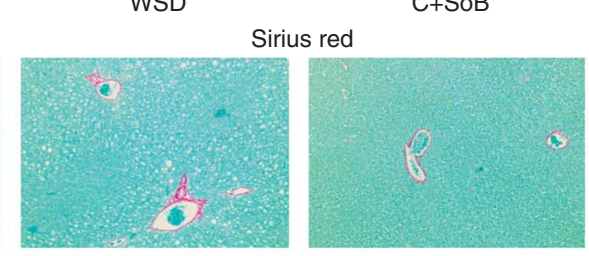

Occludin
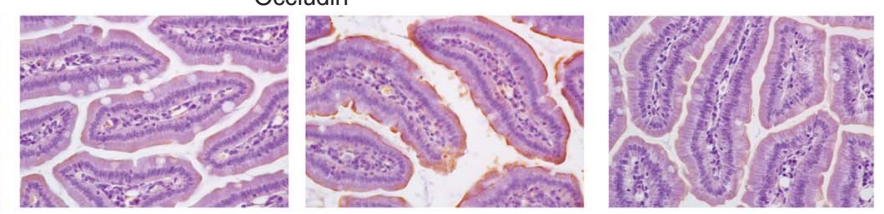

ZO-1
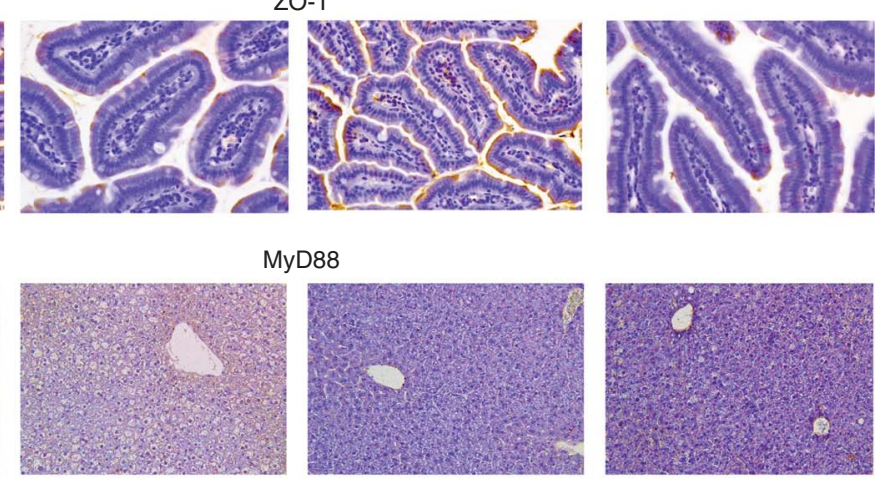

iNOS
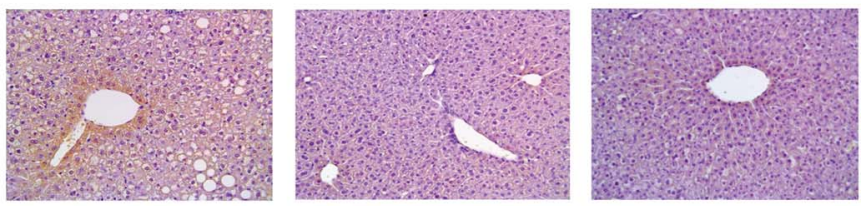

4-HNE protein adducts
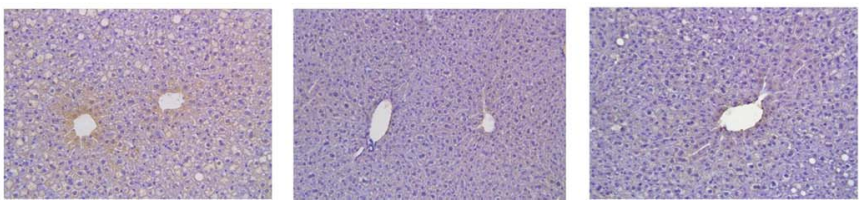

Fig. 2. Representative photomicrographs of stainings in liver and in duodenum of mice fed a Western-style diet (WSD) or control diet (C) \pm sodium butyrate (SoB). (a) Sirius red staining of liver sections (100x). (b) Occludin and (c) zonula occludens 1 (ZO-1) staining of duodenum sections (both 400x). Staining of (d) myeloid differentiation primary response gene 88 (MyD88), (e) inducible nitric oxide synthase (iNOS) and (f) 4-hydroxynonenal (4-HNE) protein adducts of liver sections (all 200x).

\section{Discussion}

Up to now, life-style- or pharmaceutical-based interventions aiming to prevent the development but even more so the progression of NAFLD are still limited. Animal-based models resembling alterations found in patients with simple steatosis as well as NASH have been found to be useful tools to investigate possible molecular mechanisms involved in the development and progression of the disease and to evaluate new potential therapeutic and preventive strategies ${ }^{(21)}$. In the present study, a mouse model using a liquid fructose-enriched WSD was used to induce not only the early metabolic but also molecular changes associated with the development of NASH (e.g. steatosis, inflammation and insulin resistance). Indeed, chronic feeding of this type of diet produced pathological changes in the liver and also at the level of the intestine that resembles many of the early alterations found in humans with beginning $\mathrm{NASH}^{(1,21,22)}$. Despite these similarities with the human situation, it needs to be emphasised that chronic intake of a pair-fed liquid diet containing $50 \mathrm{E} \%$ fructose, $25 \mathrm{E} \%$ fat and $0.155 \%$, w/w cholesterol by no means mimics all alternations found in humans with NAFLD; however, this kind of dietary model offers the possibility to study molecular mechanisms underlying the development of NASH. Furthermore, as dietary intake of animals can be adjusted between groups, this kind of model can also serve to determine efficacy of nutritional supplements or drugs in a more accurate way as differences in energy intake associated with different treatments can be accounted for. Here, 
(a)

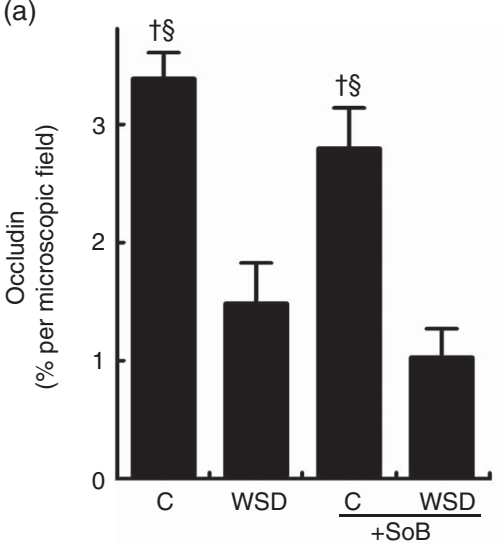

(d)

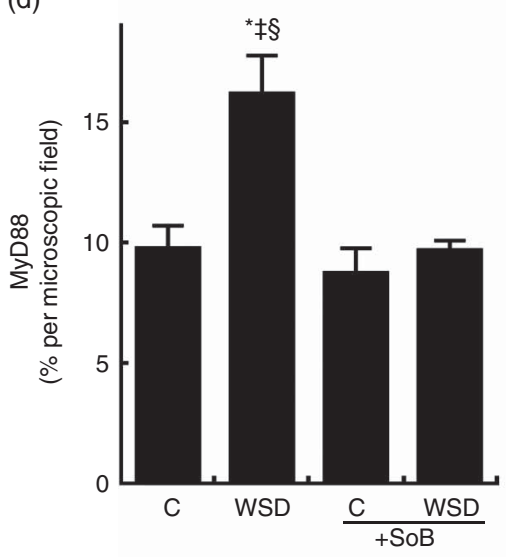

(g)

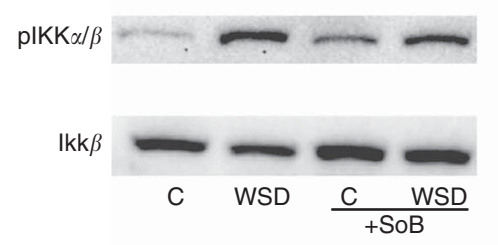

(b)

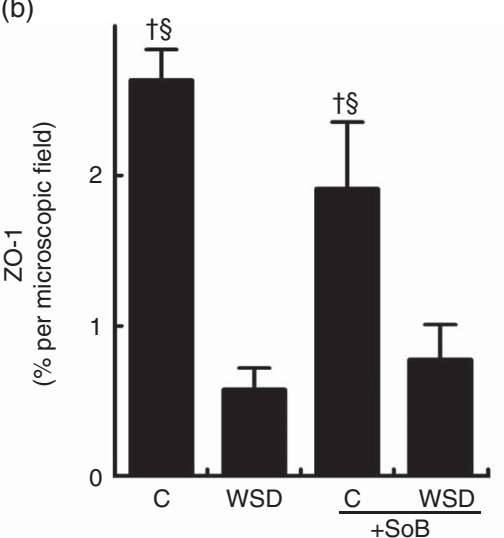

(e)

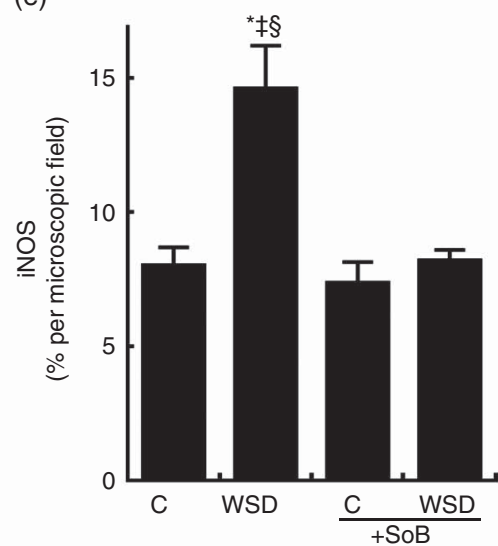

(f)

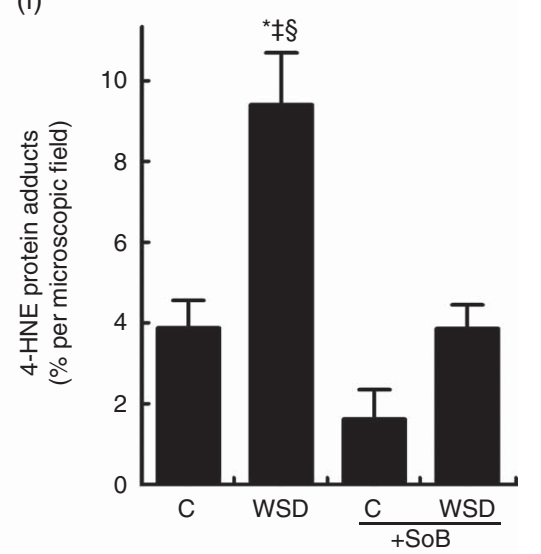

(c)

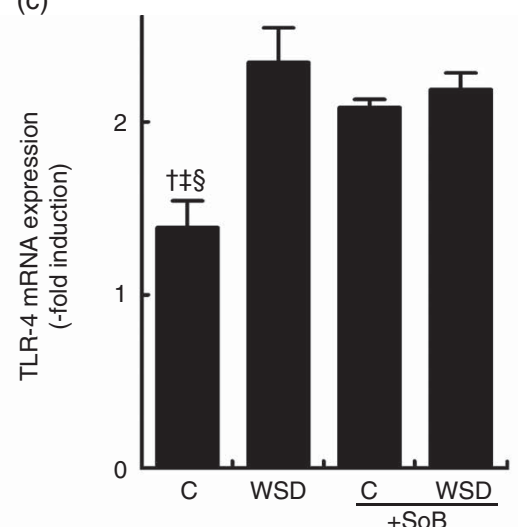

(h)

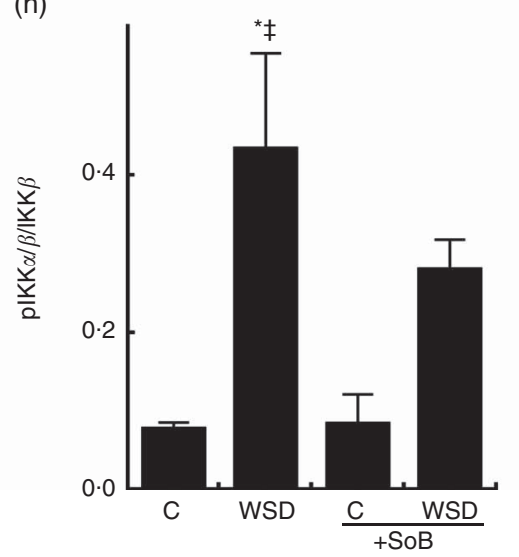

Fig. 3. Effect of the oral supplementation of sodium butyrate (SoB) on tight junction proteins in duodenum, the toll-like receptor 4 (TLR-4)-dependent signalling cascade and markers of lipid peroxidation as well as the phosphorylation status of inhibitor of NF- $k B$ kinase subunit a and $\beta$ (plKKa/ $\beta$ ) in the liver of Western-style diet (WSD)- and control diet (C)-fed mice. Densitometric analysis of immunohistochemical staining for (a) occludin, (b) zonula occludens 1 (ZO-1), (d) myeloid differentiation primary response gene 88 (MyD88), (e) inducible nitric oxide synthase (iNOS) and (f) 4-hydroxynonenal (4-HNE) protein adducts. (c) Expression of TLR-4 mRNA in liver, normalised to eukaryotic translation elongation factor 2 mRNA expression. Representative pictures ( $\mathrm{g}$ ) and densitometric analysis (h) of western blots of $(\mathrm{plKKa} / \beta)$ normalised to IKK $\beta$. Values are means, with their standard errors represented by vertical bars. ${ }^{*}<0.05$ compared with mice fed control diet, $\dagger P<0.05$ compared with mice fed WSD, $¥ P<0.05$ compared with mice fed concomitantly control diet and SoB $(0.6 \mathrm{~g} \mathrm{SoB} / \mathrm{kg}$ body weight/d), $§ P<0.05 \mathrm{compared}$ with mice fed concomitantly WSD and SoB $(0.6 \mathrm{~g} \mathrm{SoB} / \mathrm{kg}$ body weight/d).

this dietary model was used to determine whether an oral supplementation of SoB can protect mice from the development of NASH. Despite a similar intake of energy and weight gain in all feeding groups and no differences in ALT plasma levels, mice fed a WSD supplemented with SoB were markedly protected from the development of NASH. Indeed, these mice displayed less macrovesicular fat accumulation and almost no inflammatory foci in their livers. However, animals fed the WSD regardless of additional treatments did not yet display any marked histological signs of fibrosis in their livers, and TGF- $\beta 1$ mRNA expression was also only slightly increased. Indeed, it was shown in a recently published study that mice fed a WSD in 
(a)

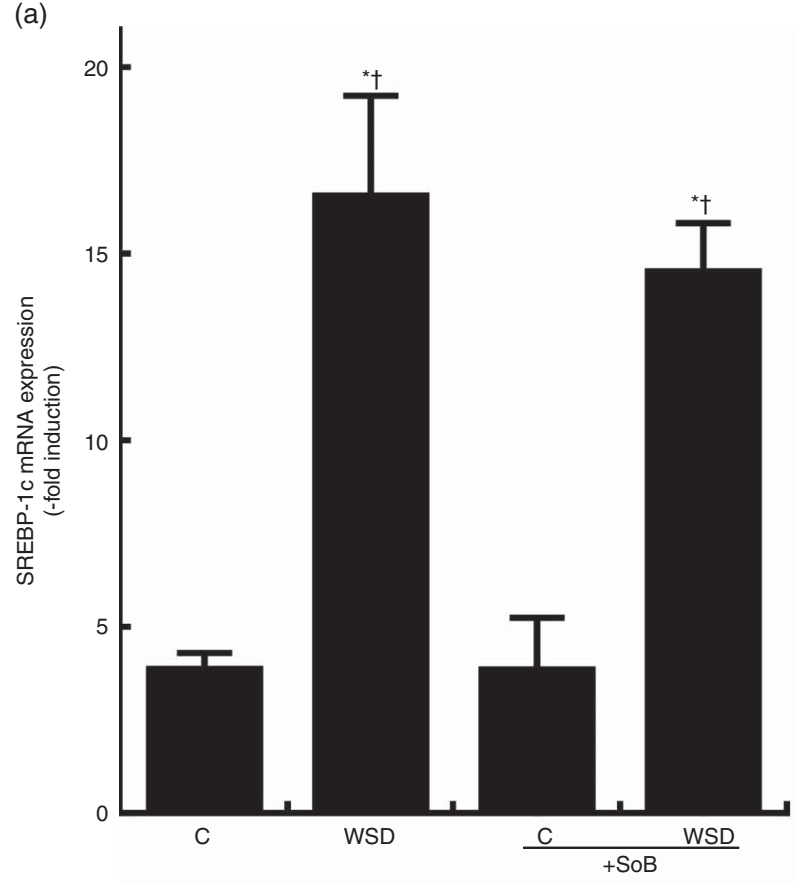

(b)

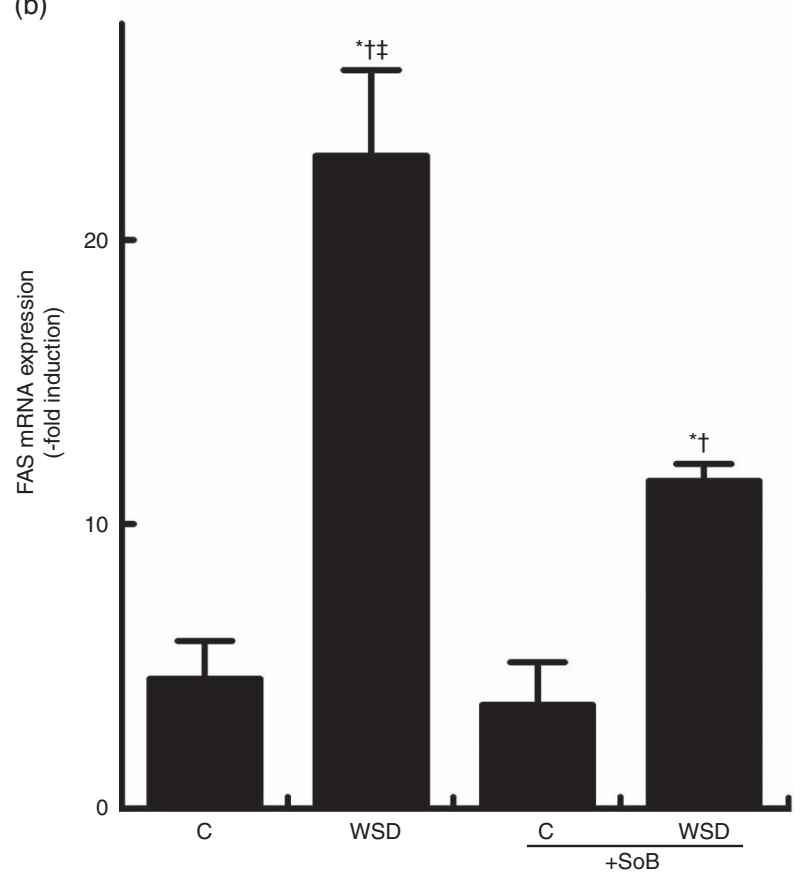

Fig. 4. Effect of the oral supplementation of sodium butyrate (SoB) on markers of lipogenesis in livers of Western-style diet (WSD)- and control diet (C)-fed mice. Expression of (a) sterol regulatory element-binding protein 1c (SREBP-1C) mRNA and (b) fatty acid synthase (FAS) mRNA in liver of WSD-fed mice. Expression of SREBP-1C and FAS was normalised to 18S. Values are means, with their standard errors represented by vertical bars. * $P<0.05$ compared with mice fed control diet, $\dagger P<0.05$ compared with mice fed concomitantly control diet and SoB $(0.6 \mathrm{~g} \mathrm{SoB} / \mathrm{kg}$ body weight $/ \mathrm{d})$, $¥ P<0.05$ compared with mice fed concomitantly WSD and SoB $(0.6 \mathrm{~g} \mathrm{SoB} / \mathrm{kg}$ body weight/d).

combination with drinking water enriched with high-fructose maize syrup for 16 weeks only showed mild signs of fibrosis ${ }^{(23)}$, suggesting that feeding time in the present study might not have been long enough yet to induce fibrotic changes in the liver. In line with the histological findings with regard to fat accumulation and inflammation, a number of neutrophils and F4/80-positive cells as well as expression of CCL-2 mRNA and to a lesser extend protein levels of TNF- $\alpha$ and phosphorylation of IKK $\beta$ were also only markedly higher in livers of mice fed the WSD alone. These findings are in line with those of others, suggesting that the progression of hepatic injury to later stages - for example, NASH and fibrosis - largely depends on a CCL-2/CCr2 (chemokine (C-C motif) receptor 2) -dependent recruitment of 'inflammatory' Ly-6Chi expressing monocytes into the liver ${ }^{(24)}$. Results of the in vitro studies of Ohira et al. ${ }^{(25)}$ indicate that butyrate may reduce $C C L-2$ and $T N F-\alpha$ expression. In line with our findings, results of others also suggest that the hepatoprotective effects of butyrate might at least in part stem from an inhibition of the NF- $\kappa \mathrm{B}$ signalling cascade ${ }^{(7)}$. In contrast, the induction of the expression of $I L-1 \beta$ found in WSD-fed mice was not attenuated in mice fed a WSD supplemented with SoB; however, supplementation of SoB per se seemed to have altered the expression of this pro-inflammatory cytokine in the liver. Indeed, expression of $I L-1 \beta$ in livers of controls fed SoB was almost at the level of that found in both WSD-fed groups. In line with these findings, the number of CD $8 \alpha$-positive cells shown to be recruited to the liver by IL- $1 \beta$-dependent mechanisms ${ }^{(26,27)}$ was also not only higher in the liver of WSDfed mice but also in controls fed SoB. However, no signs of inflammation were seen in livers of these control mice. In in vitro studies of Ohira et al. ${ }^{(28)}$, it was shown that an incubation of macrophages with butyrate especially in the presence of endotoxin is associated with an increased expression of $I L-1 \beta$. Furthermore, Mattace et $a l^{(6)}$ also reported a nonsignificant approximately 2 -fold increase in $I L-1 \beta$ expression and cyclo-oxygenase-2 (COX-2) protein levels in livers of control rats fed a diet enriched with butyrate $(20 \mathrm{mg} / \mathrm{kg}$ body weight). However, similar to our own findings, in spite of the induction of $I L-1 \beta$ mRNA and COX-2 protein levels, Mattace et al. also reported that an oral supplementation of SoB protected rats from the development of a high-fat diet-induced NAFLD. In accordance with our findings, the protective effects in this study were predominantly associated with a protection against the development of hepatic inflammation rather than lipid accumulation ${ }^{(6)}$. Further studies are needed to unravel molecular mechanisms involved in the anti-inflammatory effects of butyrate in spite of the induction of some pro-inflammatory cytokines and increased number of $\mathrm{CD} 8 \alpha$-positive cells found in the liver when SoB is supplemented. The lack of differences in ALT levels between groups found in the present study could be attributed to the fact that mice only displayed early signs of NASH and no overall impaired glucose intolerance. Indeed, it has recently been suggested that adipose tissue insulin resistance, high liver TAG content and low plasma adiponectin are major factors in the elevation of plasma aminotransferase levels in patients with NAFLD, although hepatic insulin resistance seems to be of lesser importance in this respect ${ }^{(29)}$. In the present study, blood glucose levels were similar between groups, whereas, in line with earlier findings of our group, expression of $I R$ in liver was slightly lower in livers of mice fed a WSD, indicating that mice suffered from hepatic insulin resistance $^{(10)}$. In contrast, in livers of mice concomitantly fed a 
WSD and SoB, IR expression was markedly higher than that in those only fed a WSD, suggesting that the supplementation of SoB protected mice from the development of hepatic insulin resistance. Interestingly, expression of IRS-1 was per se lower in livers of mice fed SoB regardless of the diet fed, which suggests that SoB may alter insulin signalling in the liver independent of other dietary factors. These findings need to be further investigated in future studies. However, contrary to the findings in the groups not receiving $\mathrm{SoB}$, expression of $I R S-1$ in the liver did not differ between controls and WSD-fed mice. These findings are in line with those of other groups showing that supplementation of SoB may modulate insulin signalling and thereby may protect against the development of insulin resistance ${ }^{(30,31)}$. Indeed, it has recently been shown that treating chicken with $0.25 \mathrm{~g} / \mathrm{kg}$ body weight SoB resulted in a marked decrease in protein levels of the IR $\beta$ subunit in the liver and overall systemic insulin sensitivity ${ }^{(31)}$. Furthermore, in line with the findings of our study for the liver, it has also been shown in mice fed a high-fat diet, that supplementation of $5 \%, w / w$ SoB protected animals markedly from the development of insulin resistance ${ }^{(30)}$. Taken together, our data suggest that SoB supplementation in spite of the induction of some pro-inflammatory markers in the liver may protect mice from the development of NASH and hepatic insulin resistance.

The protective effect of a sodium butyrate supplementation is associated with a protection of mice from the induction of the toll-like receptor 4 signalling cascade, lipid peroxidation and the induction of fatty acid synthase in the liver.

It has been repeatedly suggested by not only the results of human studies of our own lab but also those of other groups that an increased translocation of bacterial endotoxin and activation of TLR-dependent signalling cascades in the liver may be involved in the development of NAFLD ${ }^{(32-34)}$. Results of recent studies suggest that $\mathrm{SoB}$ may exert its protective effect on the liver and maybe also on metabolic alterations through its effects on intestinal barrier function ${ }^{(3,35,36)}$. Furthermore, we and others showed before that the development of NAFLD is associated with a loss of tight junction proteins in the upper parts of the small intestine, increased bacterial endotoxin levels in the portal vein, the elevated expression of TLR in the liver and with the activation of dependent signalling cascades ${ }^{(14,33)}$. It was further shown that a protection against the loss of tight junction proteins in the upper parts of the small intestine is associated with lower endotoxin levels and a protection of the liver from the development of NAFLD ${ }^{(11,37)}$. However, in the present study, protein levels of the tight junction protein occludin and ZO-1 were both lower in mice fed a WSD regardless of additional treatments, suggesting that protective effects of the supplementation of SoB found in the present study did not primarily stem from a protection against the loss of intestinal integrity. Differences between the results of the present study and those of other groups might have resulted from differences in the aetiology of liver disease ${ }^{(9,38)}$, for example, acute alcohol-induced liver damage and I/R, respectively, $v$. long-term feeding of a WSD and animal species used (rats $v$. mice). Furthermore, Cresci et $a l .{ }^{(9)}$ fed tributyrin in their experiments, and Endo et $a l .{ }^{(39)}$ treated animals with a butyrateproducing probiotics, whereas in the present study mice were fed SoB. Results of the present study suggest that the protective effects that were found stem from an attenuation of the induction of TLR-4-dependent signalling cascade and herein especially the induction of iNOS and an increased lipid peroxidation (e.g. formation of 4-HNE protein adducts) found in livers of mice fed a WSD. Indeed, it was shown before that iNOS is critical in regulating not only NF- $\kappa \mathrm{B}$-depending signalling cascades in the development of NAFLD but also the expression of the TLR- 4 adaptor protein MyD88 ${ }^{(20)}$. In line with our findings, Qiao et al. ${ }^{(7)}$ showed that the protective effects of SoB treatment against hepatic $\mathrm{I} / \mathrm{R}$ damage were associated with a protection against the activation of NF- $\kappa \mathrm{B}$-depending signalling pathways in Kupffer cells. Mattace et al. ${ }^{(6)}$ also found that insulin resistance and NAFLD were associated with a marked attenuation of the induction of NF- $\kappa \mathrm{B}$-depending signalling cascades in the liver; however, in this study the protective effect of the butyrate supplementation was associated with a protection against the induction of TLR- 4 . Differences between the findings of Mattace et al. ${ }^{(6)}$ and our study might have resulted from differences in experimental setups - for example, high-fat diet fed to rats $v$. WSD fed to mice. However, Mattace et al. ${ }^{(6)}$ also found a marked protection of animals against the induction of iNOS in the liver. Results of Endo et al. ${ }^{(39)}$ further indicated that a dietary supplementation of butyrate-producing probiotics probably resulting in increased formation of butyrate in the intestine attenuates the induction of $S R E B P-1 C$ mRNA in the liver. In our study, despite not affecting mRNA expression of $S R E B P-1 C$ in livers of WSD-fed mice, treatment with SoB significantly attenuated the induction of FAS mRNA expression in the liver. Differences between the present study and that of Endo et $a{ }^{(39)}$ might have resulted from differences in study design (e.g. WSD-induced NASH $v$. CDAA (choline-deficient L-amino-acid-defined diet)-induced NASH and oral feeding of sod $v$. feeding of butyrateproducing probiotics). Furthermore, Endo et $a l^{(39)}$ determined SREBP-1c protein levels in nuclear extracts, whereas in the present study we only determined mRNA expression. Indeed, it has been shown before that SREBP-1c is regulated not solely at the level of expression but also through cleavage of its membrane-bound precursor ${ }^{(40)}$. Therefore, it might be that the lack of response of $S R E B P-1 C$ mRNA expression in livers of $\mathrm{WSD}+\mathrm{SoB}$-fed mice despite the down-regulation of FAS mRNA expression in livers of these mice might have resulted from a regulation of this nuclear factor at the level of protein activation rather than a regulation at the level of transcription. Taken together, not only the results of our study as well as those of other groups ${ }^{(6,9)}$ suggest that butyrate may protect mice and rats from the development of liver damage; however, molecular mechanisms involved not only in the protection against the induction of iNOS and lipid peroxidation as well as in the downregulation of FAS remain to be determined.

\section{Conclusion}

Taken together, our data suggest that an oral supplementation of SoB at least partially protects mice from the development of dietary-induced NASH. Our data further suggest that these protective effects not primarily resulted from a protection 
against the enhanced intestinal permeability and associated translocation of bacterial endotoxins found in mice with NASH but rather resulted from an inhibition of the induction of iNOS and subsequently a decreased formation of reactive oxygen species and induction of dependent signalling cascades in the liver. However, additional studies are necessary to (1) further explore underlying mechanism and to (2) determine whether similar beneficial effects of an oral supplementation of SoB are found also in humans.

\section{Acknowledgements}

Alanine aminotransferase (ALT) measurement was carried out by the Centralised Diagnostic Laboratory Services of the Jena University Hospital.

The present project has been funded by Federal Ministry of Education and Research (FKZ: 01EA1305 to I. B.).

The contribution of the authors was as follows: I. B. designed research; C. J. J., C. S., A. J. E. and D. Z. conducted research; C. J. J. and D. Z. analysed data; C. J. J. and I. B. wrote paper; and I. B. had primary responsibility for the final content.

There are no conflicts of interest.

\section{Supplementary material}

For supplementary material/s referred to in this article, please visit http://dx.doi.org/doi:10.1017/S0007114515003621

\section{References}

1. Sass DA, Chang P \& Chopra KB (2005) Nonalcoholic fatty liver disease: a clinical review. Dig Dis Sci 50, 171-180.

2. Blachier M, Leleu H, Peck-Radosavljevic M, et al. (2013) The burden of liver disease in Europe: a review of available epidemiological data. J Hepatol 58, 593-608.

3. Leonel AJ \& Alvarez-Leite JI (2012) Butyrate: implications for intestinal function. Curr Opin Clin Nutr Metab Care 15, 474-479.

4. Elamin EE, Masclee AA, Dekker J, et al. (2013) Short-chain fatty acids activate AMP-activated protein kinase and ameliorate ethanol-induced intestinal barrier dysfunction in Caco-2 cell monolayers. J Nutr 143, 1872-1881.

5. Huang XZ, Li ZR, Zhu LB, et al. (2014) Inhibition of p38 mitogen-activated protein kinase attenuates butyrateinduced intestinal barrier impairment in a Caco-2 cell monolayer model. J Pediatr Gastroenterol Nutr 59, 264-269.

6. Mattace RG, Simeoli R, Russo R, et al. (2013) Effects of sodium butyrate and its synthetic amide derivative on liver inflammation and glucose tolerance in an animal model of steatosis induced by high fat diet. PLOS ONE 8, e68626.

7. Qiao YL, Qian JM, Wang FR, et al. (2014) Butyrate protects liver against ischemia reperfusion injury by inhibiting nuclear factor kappa B activation in Kupffer cells. J Surg Res 187, 653-659.

8. Sun J, Wu Q, Sun H, et al. (2014) Inhibition of histone deacetylase by butyrate protects rat liver from ischemic reperfusion injury. Int J Mol Sci 15, 21069-21079.

9. Cresci GA, Bush K \& Nagy LE (2014) Tributyrin supplementation protects mice from acute ethanol-induced gut injury. Alcohol Clin Exp Res 38, 1489-1501.

10. Spruss A, Henkel J, Kanuri G, et al. (2012) Female mice are more susceptible to nonalcoholic fatty liver disease: sex-specific regulation of the hepatic AMP-activated protein kinaseplasminogen activator inhibitor 1 cascade, but not the hepatic endotoxin response. Mol Med 18, 1346-1355.

11. Spruss A, Kanuri G, Stahl C, et al. (2012) Metformin protects against the development of fructose-induced steatosis in mice: role of the intestinal barrier function. Lab Invest 92, 1020-1032.

12. Brunt EM, Kleiner DE, Wilson LA, et al. (2011) Nonalcoholic fatty liver disease (NAFLD) activity score and the histopathologic diagnosis in NAFLD: distinct clinicopathologic meanings. Hepatology 53, 810-820.

13. Bergheim I, Guo L, Davis MA, et al. (2006) Critical role of plasminogen activator inhibitor-1 in cholestatic liver injury and fibrosis. J Pharmacol Exp Ther 316, 592-600.

14. Wagnerberger S, Spruss A, Kanuri G, et al. (2012) Toll-like receptors 1-9 are elevated in livers with fructose-induced hepatic steatosis. Br J Nutr 107, 1727-1738.

15. Bergheim I (2008) Antibiotics protect against fructose-induced hepatic lipid accumulation in mice: role of endotoxin. J Hepatol 48, 983-992.

16. Spruss A, Kanuri G, Wagnerberger S, et al. (2009) Toll-like receptor 4 is involved in the development of fructose-induced hepatic steatosis in mice. Hepatology 50, 1094-1104.

17. Kanuri G, Spruss A, Wagnerberger S, et al. (2011) Role of tumor necrosis factor alpha (TNFalpha) in the onset of fructose-induced nonalcoholic fatty liver disease in mice. J Nutr Biochem 22, 527-534.

18. Landmann M, Wagnerberger S, Kanuri G, et al. (2015) Beer is less harmful for the liver than plain ethanol: studies in male mice using a binge-drinking model. Alcohol Alcohol 50, 493-500.

19. Yang F, Wang LK, Li X, et al. (2014) Sodium butyrate protects against toxin-induced acute liver failure in rats. Hepatobiliary Pancreat Dis Int 13, 309-315.

20. Spruss A, Kanuri G, Uebel K, et al. (2011) Role of the inducible nitric oxide synthase in the onset of fructose-induced steatosis in mice. Antioxid Redox Signal 14, 2121-2135.

21. Kanuri G \& Bergheim I (2013) In vitro and in vivo models of non-alcoholic fatty liver disease (NAFLD). Int J Mol Sci 14, 11963-11980.

22. Tilg H \& Moschen AR (2010) Evolution of inflammation in nonalcoholic fatty liver disease: the multiple parallel hits hypothesis. Hepatology 52, 1836-1846.

23. Machado RA, Constantino LS, Tomasi CD, et al. (2012) Sodium butyrate decreases the activation of NF-kappa $\mathrm{B}$ reducing inflammation and oxidative damage in the kidney of rats subjected to contrast-induced nephropathy. Nephrol Dial Transplant 27, 3136-3140.

24. Tacke F \& Zimmermann HW (2014) Macrophage heterogeneity in liver injury and fibrosis. J Hepatol 60, 1090-1096.

25. Ohira H, Fujioka Y, Katagiri C, et al. (2013) Butyrate attenuates inflammation and lipolysis generated by the interaction of adipocytes and macrophages. J Atheroscler Thromb 20, 425-442.

26. Ben-Sasson SZ, Wang K, Cohen J, et al. (2013) IL-1beta strikingly enhances antigen-driven CD4 and CD8 T-cell responses. Cold Spring Harb Symp Quant Biol 78, 117-124.

27. Ben-Sasson SZ, Hogg A, Hu-Li J, et al. (2013) IL-1 enhances expansion, effector function, tissue localization, and memory response of antigen-specific CD8 T cells. J Exp Med 210, 491-502.

28. Ohira H, Fujioka Y, Katagiri C, et al. (2012) Butyrate enhancement of inteleukin-1beta production via activation of oxidative stress pathways in lipopolysaccharide-stimulated THP-1 cells. J Clin Biochem Nutr 50, 59-66.

29. Maximos M, Bril F, Portillo SP, et al. (2015) The role of liver fat and insulin resistance as determinants of plasma aminotransferase elevation in nonalcoholic fatty liver disease. Hepatology 61, 153-160. 
30. Gao Z, Yin J, Zhang J, et al. (2009) Butyrate improves insulin sensitivity and increases energy expenditure in mice. Diabetes 58, 1509-1517.

31. Matis G, Kulcsar A, Turowski V, et al. (2015) Effects of oral butyrate application on insulin signaling in various tissues of chickens. Domest Anim Endocrinol 50, 26-31.

32. Kanuri G, Ladurner R, Skibovskaya J, et al. (2015) Expression of toll-like receptors 1-5 but not TLR 6-10 is elevated in livers of patients with non-alcoholic fatty liver disease. Liver Int 35 562-568

33. Miele L, Valenza V, La TG, et al. (2009) Increased intestinal permeability and tight junction alterations in nonalcoholic fatty liver disease. Hepatology 49, 1877-1887.

34. Thuy S, Ladurner R, Volynets V, et al. (2008) Nonalcoholic fatty liver disease in humans is associated with increased plasma endotoxin and plasminogen activator inhibitor 1 concentrations and with fructose intake. J Nutr 138, 1452-1455.

35. Wang HB, Wang PY, Wang X, et al. (2012) Butyrate enhances intestinal epithelial barrier function via up-regulation of tight junction protein Claudin-1 transcription. Dig Dis Sci 57, 3126-3135.

36. Ma X, Fan PX, Li LS, et al. (2012) Butyrate promotes the recovering of intestinal wound healing through its positive effect on the tight junctions. J Anim Sci 90, Suppl. 4, 266-268.

37. Volynets V, Kuper MA, Strahl S, et al. (2012) Nutrition, intestinal permeability, and blood ethanol levels are altered in patients with nonalcoholic fatty liver disease (NAFLD). Dig Dis Sci 57, 1932-1941.

38. Liu B, Qian J, Wang Q, et al. (2014) Butyrate protects rat liver against total hepatic ischemia reperfusion injury with bowel congestion. PLOS ONE 9, e106184.

39. Endo H, Niioka M, Kobayashi N, et al. (2013) Butyrateproducing probiotics reduce nonalcoholic fatty liver disease progression in rats: new insight into the probiotics for the gut-liver axis. PLOS ONE 8, e63388.

40. Wang X, Sato R, Brown MS, et al. (1994) SREBP-1, a membrane-bound transcription factor released by sterolregulated proteolysis. Cell 77, 53-62. 